\title{
Update from the Insulin Pump Network UK
}

\author{
EMMA G WILMOT
}

Insulin pump therapy and continuous glucose monitoring (CGM) are recognised as effective evidence-based interventions for a specific group of patients with type 1 diabetes, as outlined in the NICE [TA151] guidance. However, access to these technologies can vary across the country. We have a duty to work towards equitable access to diabetes technologies across the UK to drive down the variation in outcomes for people living with type 1 diabetes. With this in mind, the Association of British Clinical Diabetologists (ABCD) has supported the development of the Insulin Pump Network UK (IPN-UK). The IPN-UK committee is composed of a group of UK consultants with an interest in type 1 diabetes and insulin pump therapy. During this first year we have prioritised (1) education and support for insulin pump teams through educational events; (2) supporting the National Diabetes Insulin Pump Audit, including the development of a service level audit for 2017; and (3) promoting patient access to diabetes technologies.

\section{Education and support for insulin pump teams}

The IPN-UK was successfully launched in April 2016 at the inaugural meeting in Manchester. Demand for places at this meeting was high, with 140 delegates present and a further 30 on the waiting list. From the delegates at the IPN-UK meeting, one quarter were not confident in the effective use of insulin pumps and one third were not confident to discuss the pros and cons of flash glucose monitoring versus CGM, highlighting the need and desire for ongoing training and education. As such, the IPN-UK plan to run a further four events over the next 12 months. IPN-UK team days are planned for January: in Derby on 6th January led by Peter Hammond and myself and in London on 10th January led by Pratik Choudhary, each with an audience of about 60 delegates. The annual IPN-UK meeting, with $>100$ delegate spaces, will be held in Belfast on 10th May, the day prior to the ABCD Spring meeting. Later in the year we also plan to deliver a one-day insulin pump course aimed at healthcare professionals who are relatively new to insulin pump therapy. More details are available at www.ipnuk.co.uk.

Derby Teaching Hospitals NHS Foundation Trust

Address for correspondence: Dr Emma G Wilmot

Chair Insulin Pump Network UK, Derby Teaching Hospitals NHS

Foundation Trust, Royal Derby Hospital, Uttoxeter New Rd,

Derby DE22 3NE, UK

Tel: +44 (0)1332 787696

E-mail: emma.g.wilmot@gmail.com

Br J Diabetes 2016;16:149-150

http://dx.doi.org/10.15277/bjd.2016.100

\section{National Audit of Insulin Pump Therapy: patient and service level data}

Insulin pump therapy is associated with a number of benefits which include improved glycaemic control and a reduction in severe and non-severe hypoglycaemia. NICE guidance recommends the use of insulin pumps in those with an elevated $\mathrm{HbA}_{1 c}$ despite optimised multiple daily injections and/or those who experience problematic hypoglycaemia. The 2012 UK National Insulin Pump Audit identified that $6 \%$ of people with type 1 diabetes were treated with insulin pumps. Repeat audit in 2015 suggested this had increased to 12\%, although this figure varied substantially between centres (from $<5 \%$ to $>30 \%$ ) and was limited to data from 44 centres compared with 178 centres who contributed to the 2012 service level audit. 1,2 A repeat detailed service level audit is planned for 2017. This will assess key elements of type 1 diabetes services including the use of and funding for insulin pump therapy and CGM and staffing available to support those using these technologies. This will allow teams to benchmark their services in terms of the level of service provision, staffing and funding agreements which could support future business cases to further improve type 1 diabetes care.

\section{Promoting patient access to diabetes technologies}

\section{Continuous glucose monitoring (CGM)}

It is now over a year since the publication of NICE type 1 diabetes in adults [NG17] guidance which recommended CGM for those with problematic hypoglycaemia, as outlined in Box 1. ${ }^{3}$ Unfortunately, this guidance has not translated into automatic funding for those who need the technology most. While there are some Clinical Commissioning Groups (CCGs) who provide funding for CGM for patients who meet the NICE guidance, there are some who put a cap on access to CGM and some who do not fund CGM at all. This is concerning as this will lead to the development of a postcode lottery with inequitable access to diabetes technology. We do not currently understand how many CCGs have refused to fund CGM. The service level audit described above should provide some insight. In the meantime, if your CCG has refused to fund CGM or insulin pumps, please contact the IPN-UK.

\section{Freestyle Libre}

A relatively recent addition to the armamentarium of available diabetes technologies is the Freestyle Libre. This is an arm-worn sensor which measures the interstitial glucose. When the reader is used to scan the sensor, the glucose level and direction of travel is displayed. Although it displays a continuous trace of glucose data, it is not CGM. It does not alarm to alert the patient when the glucose levels 
are falling and glucose data cannot be viewed without the patient scanning the sensor.

The number of patients self-funding the Freestyle Libre has risen exponentially over the past two years. The publication by Bolinder et $a$ l in the Lancet in September highlighted a number of benefits of this system. ${ }^{4}$ In well controlled patients with type 1 diabetes there was a 38\% reduction in hypoglycaemia and improved treatment satisfaction. Despite no training in interpretation of the glucose data, the reduction in hypoglycaemia occurred within the first two weeks of therapy, before contact was made with a healthcare professional. To achieve these benefits the participants were scanning their glucose levels approximately 15 times per day, a reflection of the demands of living with well controlled type 1 diabetes. The Freestyle Libre undoubtedly brings benefits to those who choose to use it. Sadly, this use is often restricted to the most affluent individuals who can afford to self-fund. The Freestyle Libre is a very popular option with patients and the IPN-UK would support future NHS funding.

The Freestyle Libre does present some challenges for diabetes teams. The first is our ability to interpret data from the device and the second is defining the exact role it has to play in the diabetes care pathway. Unlike finger stick blood glucose monitoring, data from the Freestyle Libre cannot be recorded in a diary. To optimise therapy, healthcare professionals will need to become confident and able to assess the data from this device if we are to adequately support those who choose to use it. However, the majority of UK clinicians have limited experience of continuous glucose data interpretation and, currently, less than half of UK diabetologists download glucose data in their clinics. Options for viewing glucose data from this device include Diasend (for an annual fee) or installing the Abbott software free of charge. We recognise that data interpretation from both flash glucose monitoring and CGM is an ongoing challenge, and this will be a key aspect of the educational events delivered by IPN-UK.

In terms of the role for the Freestyle Libre in the treatment pathway, it is not CGM. It does not alarm when the glucose level drops. It has not been evaluated in patients with impaired awareness of hypoglycaemia or recurrent severe hypoglycaemia. It is likely that those with impaired awareness of hypoglycaemia are dependent on the alarms from CGM to alert them to impending hypoglycaemia. Until we have evidence from randomised controlled trials to suggest otherwise, CGM and sensor augmented pump therapy should remain the intervention of choice in this high-risk group. 3,5

\section{IPN membership}

If you have not done so already, you can register for updates from the IPN-UK at www.ipn-uk.co.uk

\section{Conclusions}

Over the coming year IPN-UK will be delivering a number of events for healthcare professionals with an interest in diabetes technologies. We will be working with the National Diabetes Audit team to
Box 1. NICE indications for continuous glucose monitoring (CGM)

Consider real-time CGM for adults with type 1 diabetes who are willing to commit to using it at least $70 \%$ of the time and to calibrate it as needed, and who have any of the following despite optimised use of insulin therapy and conventional blood glucose monitoring:

- More than one episode a year of severe hypoglycaemia with no obviously preventable precipitating cause

- Complete loss of awareness of hypoglycaemia

- Frequent (>2 episodes a week) asymptomatic hypoglycaemia that is causing problems with daily activities

- Extreme fear of hypoglycaemia

- Hyperglycaemia $\left(\mathrm{HbA}_{1 \mathrm{c}}\right.$ level $75 \mathrm{mmol} / \mathrm{mol}(9 \%)$ or higher) that persists despite testing at least 10 times a day. Continue real-time continuous glucose monitoring only if $\mathrm{HbA}_{1 \mathrm{c}}$ can be sustained at or below $53 \mathrm{mmol} / \mathrm{mol}(7 \%)$ and/or there has been a fall in $\mathrm{HbA}_{1 \mathrm{c}}$ of $27 \mathrm{mmol} / \mathrm{mol}(2.5 \%)$ or more

develop a national service level audit which, in the future, will allow us to benchmark access to insulin pumps, CGM and the level of staffing to support patients using these technologies. We also hope to develop a number of documents which will provide healthcare professionals with guidance on pump and CGM use. In the meantime, if your patients have no access to an insulin pump or CGM in your service, please do contact us.

\section{IPN sponsors}

Gold sponsors: Cellnovo, Diasend, Roche

Silver sponsors: Abbott, Animas, Medtronic (Gold from 2017), Medtrum (from 2017)

Bronze sponsors: Dexcom

\section{References}

1. The United Kingdom Insulin Pump Audit. https://www. diabetes.org.uk/ Documents/News/The_United_Kingdom_Insulin_Pump_Audit_May_201 3.pdf (accessed 27 Oct 2016).

2. HSCIC National Diabetes Insulin Pump Audit Report, 2013-15. http://www.hqip.org.uk/public/cms/253/625/19/520/National\%20Diabetes\%20Insulin\%20Pump\%20Audit\%20report\%202013-15.pdf?realName=hOfPw8.pdf?v=0 (accessed 27 Oct 2016).

3. NICE. Type 1 diabetes in adults: diagnosis and management. NICE guideline [NG17]. August 2015. https://www.nice.org.uk/guidance/ ng17? unlid=843694082201610196118 (accessed 26 Oct 2016).

4. Bolinder J, Antuna R, Geelhoed-Duijvestijn P, Kroger J, Weitgasser R. Novel glucose-sensing technology and hypoglycaemia in type 1 diabetes: a multicentre, non-masked, randomised controlled trial. Lancet. Published Online First: 9 Sept 2016.

http://dx.doi.org/10.1016/S0140-6736(16)31535-5

5. NICE. Type 1 diabetes: Integrated sensor-augmented pump therapy systems for managing blood glucose levels (The MiniMed Paradigm Veo System and the Vibe and G4 PLATINUM CGM system). Diagnostics Guidance [DG21]. February 2016. https://www.nice.org.uk/guidance/indevelopment/gid-dt22 (accessed 26 Oct 2016). 